\title{
Energy non-linearity effects in argon gaseous detectors in the region of the Ar K-absorption edge: experimental results
}

\author{
C.M.B. Monteiro, P.C.P.S. Simões, J.F.C.A. Veloso, J.M.F. dos Santos*, \\ C.A.N. Conde \\ Physics Department, University of Coimbra, Coimbra P-3004-516, Portugal
}

\begin{abstract}
The response of an argon proportional counter to X-rays with energy in the range of $1.5-8 \mathrm{keV}$ was investigated. The response to full-energy absorption events was found to follow different trends for X-ray energies below and above the argon K-absorption edge presenting a discontinuity at this energy. An abrupt decrease of $(2.2 \pm 0.3) \%$ in the detector amplitude at the K-edge $(3.203 \mathrm{keV})$ was measured, corresponding to a region within $\pm(70 \pm 10) \mathrm{eV}$ around the edge where the X-ray energy cannot be obtained unambiguously. This discontinuity corresponds to an abrupt increase of about $0.5 \mathrm{eV}$ in the argon $w$-value and results in a sudden increase in the energy resolution from about $17 \%$ to about $19 \%$. The detector response to escape peak events follows a linear trend over the entire energy range, very close to the response for full-energy absorption of X-rays with energies above the K-edge.
\end{abstract}

(C) 2003 Elsevier Science B.V. All rights reserved.

PACS: $07.85 . \mathrm{Nc} ; 29.40 . \mathrm{Cs} ; 32.80 . \mathrm{t} ; 32.80 . \mathrm{fb}$

Keywords: Argon; X-ray detectors; Non-linearity; K-edge

\section{Introduction}

Argon-based gaseous detectors are widely used in X-ray spectrometry with applications to important areas such as X-ray astrophysics and energy dispersive $\mathrm{X}$-ray fluorescence analysis [1]. Laboratory and portable systems instrumented with argon gaseous detectors have been developed for both R\&D and industrial environments. A detailed knowledge of the detector energy linearity

*Corresponding author. Tel.: + 351-239-410667; fax: + 351239-829158.

E-mail address: jmf@gian.fis.uc.pt (J.M.F. dos Santos). and energy resolution is necessary to perform an accurate data analysis.

Deviations from linearity in the energy response of xenon gaseous detectors are well documented [2-10]. Both, experimental and simulation results have shown that departure from linearity of the energy response of gaseous detectors occurs at the gas absorption edges, e.g. an abrupt decrease of about $1.5 \%$ and $0.6 \%$ in the detector amplitude at the xenon $\mathrm{L}_{\mathrm{III}}$ - and $\mathrm{K}$-absorption edges was found for different type of gaseous detectors [9].

The quantitative explanation for the discontinuities was given in Refs. [3,9] using a detailed Monte Carlo simulation model [11]. The efficiency for converting absorbed X-ray energy into 
ionisation is lower for atomic sub-shells with higher binding energies. When a new photoionisation channel becomes energetically accessible the subsequent de-excitation cascade of the photoionised atom results in a larger number of electron vacancies in the outermost sub-shells. A measurable amount of the absorbed energy can be expended in establishing the ground state of the ion with the additional vacancies. Additionally, the overall kinetic energy of the photoelectron and the other electrons emitted by the photoionised atom decreases, producing a smaller number of primary electrons during the thermalisation process. The same reasoning can be applied to explain the differences in the detector energy response to full-energy absorption and to escape peak events with the same energy $[8,10]$.

The discontinuities in the energy response for argon-filled detectors are not well documented. Jahoda and McCammon [12] investigated the response of an argon-methane proportional counter to X-rays with energy in the $99-277 \mathrm{eV}$ range and reported a discontinuity of about $12 \%$ in the detector amplitude at the $\mathrm{Ar} \mathrm{L}_{\mathrm{III}}$-edge $(246 \mathrm{eV})$. Results for the Ar K-edge ( $3.203 \mathrm{keV})$ have not yet been published, to the best of our knowledge. The argon photoionisation due to $\mathrm{X}$-ray energies around the argon K-edge has been studied [1315] and experimental results show that the average charge state increases from 2.9 to 4 and that the yields for the +5 and +6 charge states increase by a factor above 50 [14].

In the present work we experimentally investigate the response of an argon proportional counter to X-rays for both full-energy absorption and $\mathrm{Ar}$ $\mathrm{K}_{\alpha}$-fluorescence escape events with energies in the region around the Ar K-absorption edge.

\section{Experimental setup and method}

The detector used in this work was a 0460_3AR proportional counter from Metorex Oy [16]. The detector has a $2.5-\mathrm{cm}$ diameter, stainless steel cylindrical body, a $2.5-\mathrm{cm}$ diameter, $250-\mu \mathrm{m}$ thick beryllium window and is filled with argon at $4 \times 10^{5} \mathrm{~Pa}$. Through the experiment, the detector was biased at $935 \mathrm{~V}$. Detector pulses were fed through a Metorex MCPS 2194 charge preamplifier to a HP 5582A main amplifier, using shaping times of $5 \mu \mathrm{s}$. A Nucleus PCA2, 1024 multichannel analyser was used to store the pulse-height distributions. The non-linearity in the electronic chain was determined using a BNC-PB4 high precision pulse generator. The pulse amplitudes versus channel number were fitted to two straight lines, one above and one below the channel corresponding to $3.203 \mathrm{keV}$. The values of the ordinates of each line extrapolated to the channel of interest differed by less than $0.1 \%$.

The required X-ray energies were generated by exciting K-fluorescence lines in selected target elements from $\mathrm{Al}(1.74 \mathrm{keV})$ to $\mathrm{Cu}(8.0 \mathrm{keV})$. The fluorescent targets were positioned at $45^{\circ}$ to the detector axis while the radioactive source (either ${ }^{244} \mathrm{Cm}$ alpha source or a ${ }^{109} \mathrm{Cd} \mathrm{X}$-ray source) was positioned at $90^{\circ}$. A $10-\mathrm{cm}$ diameter lead collimator placed over the detector window delimited the scattered and fluorescent X-rays. The counting rate in the detector was maintained below $150 \mathrm{c} / \mathrm{s}$ in all cases, a rate sufficiently low to neglect any space charge effect inside the detector.

The measured pulse-height distributions were fitted to Prescott functions [7] superimposed on a linear background, using the grid least-squares fit method [17]. According to Ref. [7], the Prescott function fits proportional counter peaks significantly better than the Gaussian function, which cannot reproduce the slight skewness characteristic of the pulse-height distributions resulting from charge multiplication. Pulse-height distributions from characteristic fluorescence and respective escape peaks of elements above $\mathrm{Ca}$ were fitted to two Prescott functions corresponding to the $\mathrm{K}_{\alpha^{-}}$ and $\mathrm{K}_{\beta}$-fluorescence, while for lighter elements only a single $\mathrm{K}_{\alpha, \beta}$ peak was considered.

The peak centroid and the respective energy resolution were monitored as a function of the Xray energy. By measuring these discrete energies we obtain the integral non-linearity in the detector response, as opposed to the differential and integral non-linearities that could be obtained using a continuously tuneable X-ray source, such as synchrotron radiation. However, this method has been used to measure the non-linearity in the response of a xenon gaseous detector around the 
Xe L- and K-edges [5,6] and of an HPGe solidstate detector around the Ge K-edge $[18,19]$ and presented results similar to those obtained with other methods [9]. Additionally, as a single discontinuity exists at the K-edge, the analysis performed with this method is valid for the whole covered region, except for a small region within the range of less than $100 \mathrm{eV}$ around the $\mathrm{K}$-edge, as can be inferred by the studies on the argon photoionisation due to X-ray energies around the argon K-edge [13-15].

Two independent runs were performed and, for each run, different gains were used in the main amplifier, corresponding to about $6.5-$ and $7.2-\mathrm{eV} /$ channel in the MCA, respectively. For each run, the system stability was determined by monitoring the pulse-height distribution for a 2-mm collimated $5.9-\mathrm{keV}$ X-ray beam from a ${ }^{55} \mathrm{Fe}$ radioactive source, with the $\mathrm{K}_{\beta}$-line filtered with of a chromium foil. In this manner, the centroid position uncertainty was found to be less than 1 and 0.7 channels, respectively.

\section{Experimental results and discussion}

In Fig. 1(a) we have plotted the $\mathrm{K}_{\alpha}$ peak centroids as a function of the energy, for run 2 , with a least-squares fit of straight lines to each set of data below and above the argon K-edge threshold. The region around the $\mathrm{K}$-edge is magnified in Fig. 1(b). While the detector response is found to be approximately linear below and above the K-edge, a clear discontinuity of about $2.4 \%$ is observed at the $\mathrm{K}$-edge. This discontinuity results in a region within about $\pm 76 \mathrm{eV}$ around the edge where the X-ray energy cannot be obtained unambiguously, since there are two distinct values for the X-ray energy corresponding to a given channel. Figs. 2(a) and (b) present for run 1 the same plot as Fig. 1, together with the centroids from the $\operatorname{Ar} \mathrm{K}_{\alpha}$-fluorescence escape peaks.

While the measured discontinuity for the fullenergy absorption events is about $1.9 \%$, corresponding to a $\sim \pm 62 \mathrm{eV}$ region around the $\mathrm{K}$-edge, the detector response to $\mathrm{Ar} \mathrm{K}_{\alpha}$-escape peaks follows a linear behaviour over the entire range of energies. As shown in Fig. 2, the detector
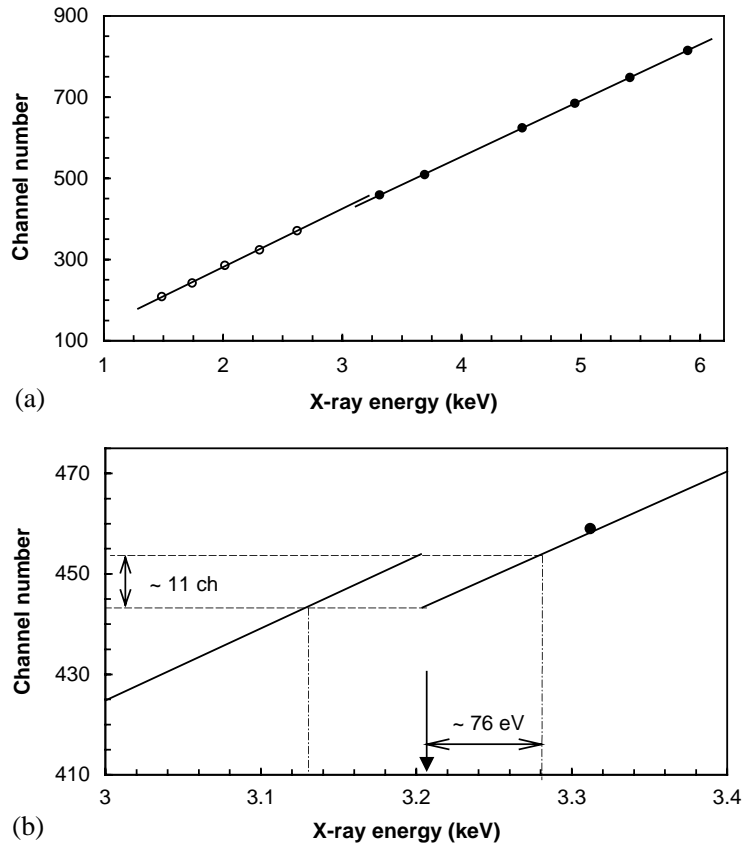

Fig. 1. (a) MCA peak centroids as a function of the X-ray energy, for the full-energy absorption of the $\mathrm{K}_{\alpha}$-fluorescence, for run 2, with a least-squares fit of straight lines to each set of data, below and above the argon K-edge threshold. (b) The least-squares fits to the data in the region of the argon K-edge.

response to escape peak events closely follows the response to full-energy absorption of X-rays with energies above the K-edge, somewhat above the experimental errors. This behaviour is similar to what was found for xenon detectors $[8,10]$ and is justified considering that both type of events result from X-ray interactions where the photoionisation preferentially takes place in the atom K-shell.

Figs. 1(a) and 2(a) show a somewhat steeper slope for the linear response to full-energy absorption events below the K-edge when compared to that of events above the K-edge. This effect is also observed for xenon detectors around the K-edge [9].

Assuming a detector gain, $G$, the centroid channel number, $A$, and the average number of primary electrons, $N$, produced by the X-rays with energy $E_{\mathrm{x}}$, are related by $A=G N$. The $w$-value, i.e. the average energy to produce an electron/ion 


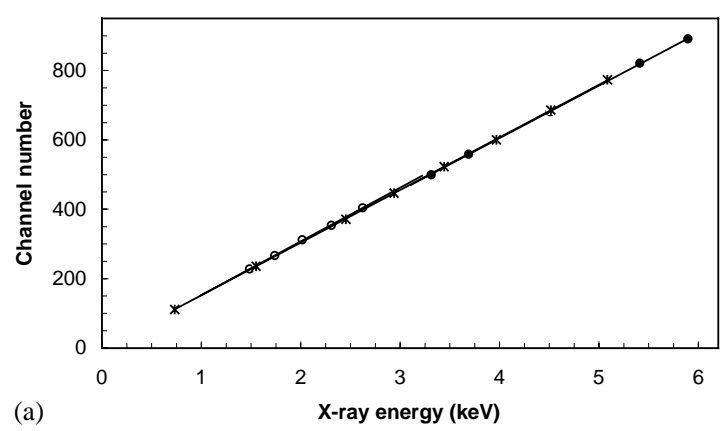

(a)

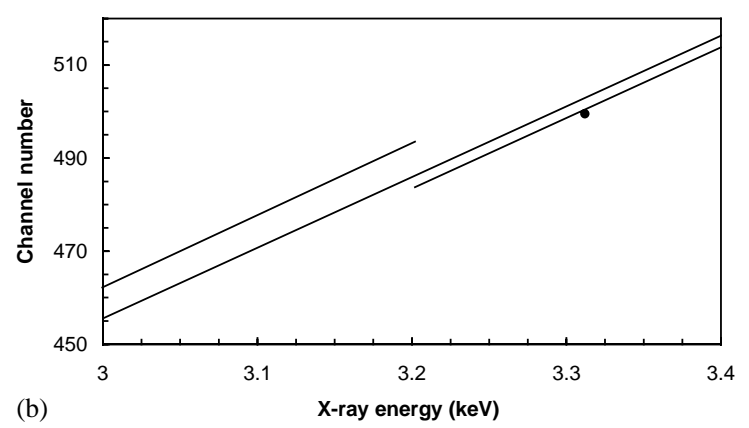

Fig. 2. (a) MCA peak centroids as a function of the energy absorbed in the detector, for full-energy absorption events $(O$, -) and for the $\mathrm{Ar} \mathrm{K}_{\alpha}$-fluorescence escape events (*) resulting from the $\mathrm{K}_{\alpha}$-fluorescence for run 1 . The solid lines are the leastsquares fits to each set of data: full-energy absorption events with energies below and above the argon $\mathrm{K}$-edge, $\operatorname{Ar} \mathrm{K}_{\alpha^{-}}$ fluorescence escape events. (b) The least-squares fits to the data in the region of the $\mathrm{K}$-edge.

pair, is given by

$w=\frac{E_{\mathrm{x}}}{N}=G \frac{E_{\mathrm{x}}}{A}$

being proportional to the $E_{\mathrm{x}} / A$ ratio. Fig. 3 presents the $w$ relative behaviour, as a function of the energy deposited in the detector, for the fullenergy absorption events below and above the Kedge, and for the $\mathrm{Ar} \mathrm{K}_{\alpha}$-fluorescence escape events. Absolute values for $w$ were obtained normalizing the relative value at $5.895-26.2 \mathrm{eV}$ [1]. Although the experimental fluctuations in the relative value of $w$ are significant, in particular for the data of the full-energy absorption events with energies below the K-edge, a sharp rise of about $2 \%(\sim 0.5 \mathrm{eV})$ is noticeable at the K-edge. The observed slow decrease with the X-ray energy, for the data above the K-edge, departs from the characteristic behaviour of an ideal detector, an

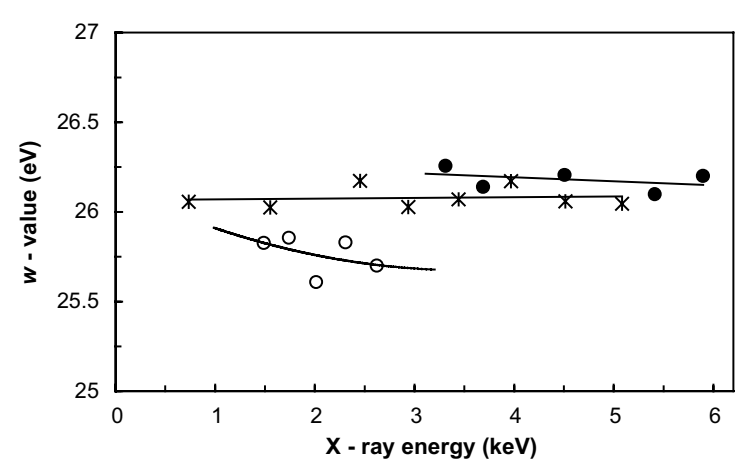

Fig. 3. Experimental results for the argon $w$-value as a function of the energy deposited in the detector for the full-energy absorption events below $(\bigcirc)$ and above $(\bullet)$ the argon $\mathrm{K}$ edge, and for the $\mathrm{Ar} \mathrm{K}_{\alpha}$-fluorescence escape events ( $\left.\boldsymbol{*}\right)$. Absolute values of $w$ were obtained normalizing the relative experimental value at $5.895-26.2 \mathrm{eV}$. The solid lines serve only to guide the eye.

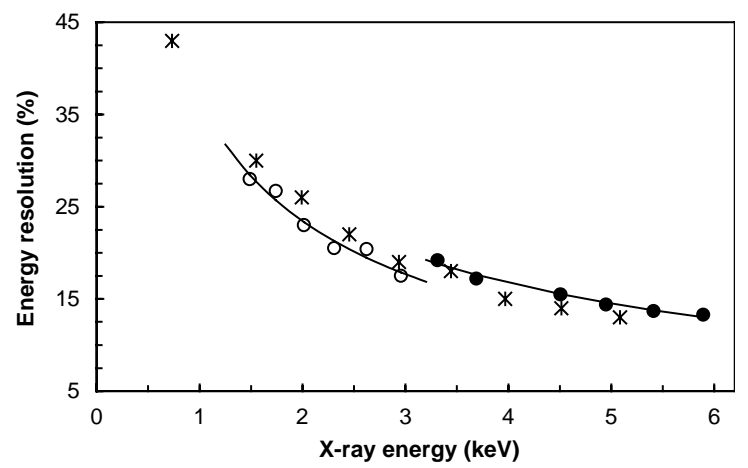

Fig. 4. Detector energy resolution (relative FWHM) as a function of the energy deposited in the detector for the fullenergy absorption events below $(\bigcirc)$ and above $(\bullet)$ the argon $\mathrm{K}$-edge, and for the $\mathrm{Ar} \mathrm{K}_{\alpha}$-fluorescence escape events (*). The solid lines represent the least-squares fits to $E_{\mathrm{x}}^{-1 / 2}$ for each set of full-energy absorption events.

effect that was also observed in xenon detectors [9]. However, the deviation observed in real detectors does not invalidate the measured results of their response to X-rays, as shown for the xenon case.

In Fig. 4 we depict the detector energy resolution (relative FWHM) for the given experimental conditions, as a function of the peak energy.

These results clearly confirm a discontinuous behaviour of the detector response to full-energy 
absorption events at the K-edge: the energy resolution degrades by an absolute value of about $2 \%$. The detector energy resolution for escape peak events follows a continuous trend, denoting a response that is different from those corresponding to full-energy absorption events.

\section{Conclusions}

We investigated the response of an argon proportional counter to $\mathrm{X}$-rays with energies in the $1.5-8 \mathrm{keV}$ range. Both full-energy absorption events and argon $\mathrm{K}_{\alpha}$-fluorescence escape events have been monitored. The results for the peak centroids, energy resolution and $w$-value show a discontinuity of the detector response for fullenergy absorption events, while no discontinuity is observed in the detector response for the $\mathrm{Ar} \mathrm{K}_{\alpha^{-}}$ fluorescence escape events. We have measured an abrupt decrease of $(2.2 \pm 0.3) \%$ in the detector amplitude at the K-edge, corresponding to a region within $\pm(70 \pm 10) \mathrm{eV}$ around the edge where the X-ray energy cannot be obtained unambiguously. This discontinuity corresponds to an abrupt increase of about $0.5 \mathrm{eV}$ in the $w$-value. The discontinuity in the detector energy resolution was found to have a degradation of about $2 \%$, absolute value, for full-energy absorption peaks. The detector response to escape peak events follows a linear trend very close to that of fullenergy absorption events for X-rays with energies above the K-edge.

\section{Acknowledgements}

The work was carried out in the laboratory of the Atomic and Nuclear Instrumentation Group of the Instrumentation Centre (Unit 217/94) of Departamento de Física, Universidade de Coimbra. Support is acknowledged from projects CERN/FIS/43787/01 and POCTI/ 43527/99. JMF dos Santos acknowledges travel support from Fundação Luso-Americana para o Desen- volvimento and Fundação Calouste Gulbenkian, Lisbon. JFCA Veloso acknowledges travel support from Fundação Calouste Gulbenkian and FCT, Lisbon.

\section{References}

[1] G.F. Knoll, Radiation Detection and Measurements, 3rd Edition, Wiley, New York, 2000.

[2] P. Lamb, G. Manzo, S. Re, et al., Astrophys. Space Sci. A 136 (1987) 369.

[3] F.P. Santos, T.V.H.T. Dias, A.D. Stauffer, C.A.N. Conde, Nucl. Instr. and Meth. A 307 (1991) 347.

[4] H. Tsunemi, K. Hayashida, K. Torii, et al., Nucl. Instr. and Meth. A 336 (1993) 301.

[5] J.M.F. dos Santos, C.A.N. Conde, A.C.S.S.M. Bento, Nucl. Instr. and Meth. A 324 (1993) 611.

[6] J.M.F. dos Santos, R.E. Morgado, L.M.N. Tavora, C.A.N. Conde, Nucl. Instr. and Meth. A 350 (1994) 216.

[7] Budtz-Jorgensen, C. Olesen, H.W. Schnopper, T. Lederer, F. Scholze, G. Ulm, Nucl. Instr. and Meth. A 367 (1995) 83.

[8] T.H.V.T. Dias, F.P. Santos, J.M.F. dos Santos, et al., IEEE Trans Nucl. Sci. NS-43 (1996) 1452.

[9] T.H.V.T. Dias, J.M.F. dos Santos, P.J.B.M. Rachinhas, F.P. Santos, C.A.N. Conde, A.D. Stauffer, J. Appl. Phys. 82 (1997) 2742.

[10] G. Zavattini, M. Feroci, C. Budtz-Jorgensen, et al., Nucl. Instr. and Meth. A 401 (1997) 206.

[11] T.H.V.T. Dias, F.P. Santos, A.D. Stauffer, C.A.N. Conde, Phys. Rev. A 48 (1993) 2887.

[12] K. Jahoda, D. McCammon, Nucl. Instr. and Meth. A 272 (1988) 800

[13] J. Doppelfeld, N. Anders, B. Esser, F. von Busch, H. Scherer, S. Zinz, J. Phys. B 26 (1993) 445.

[14] H.G. Berry, Y. Azuma, P.L. Cowan, D.S. Gemmell, T. LeBrun, M. Ya Amusia, Nucl. Instr. and Meth. B 98 (1995) 25.

[15] D.V. Morgan, R. Barttlett, M. Sagurton, Phys. Rev. A 51 (1995) 2939.

[16] Metorex International Oy, P.O. Box 85, FIN-02631 ESPOO, Finland.

[17] P.R. Bevington, Data Reduction and Error Analysis for the Physical Sciences, McGraw-Hill, New York, 1969, pp. 208-214.

[18] J.M.F. dos Santos, C.M.B. Monteiro, R.E. Morgado, C.A.N. Conde, Appl. Radiat. Isot. 53 (2000) 739.

[19] J.M.F. dos Santos, C.M.B. Monteiro, Appl. Radiat. Isot. 55 (2001) 331 